Classification

Physics Abstracts

$07.80-87.64$

\title{
Are electron and near-field microscopies complementary?
}

\author{
Etienne Delain, Eric Le Cam, Agnès Barbin-Arbogast and Alain Fourcade
}

Laboratoire de Microscopie cellulaire et moléculaire, URA 147 du Centre National de la Recherche Scientifique (CNRS), Institut Gustave-Roussy, Rue Camille Desmoulins, F-94805 Villejuif Cedex, France

(Received July 4; accepted October 26, 1994)

\begin{abstract}
Résumé . - Au vu du développement considérable de l'imagerie des macromolécules biologiques à l'aide des microscopes à champ proche, il est rappelé que ce type d'observations d'objets biologiques pose encore de réels problèmes, tant au niveau méthodologiques pour la préparation des échantillons, qu'au niveau de l'interprétation des images. Une approche interdisciplinaire est recommandable, qui utiliserait conjointement les méthodes traditionnelles de la biophysique et de la biochimie, et celles, relativement mieux établies, de la microscopie électronique. La microscopie en champ proche possède cependant des possibilités uniques d'observation et d'analyse des objets biologiques, qui ne sont pas accessibles en microscopie électronique, et qui confèrent à ces nouvelles microscopies une nouvelle dimension à l'imagerie en biologie. Lillustration fournie donne des exemples des possibilités et des limitations de la microscopie électronique, et propose quelques domaines dans lesquels la complémentarité des différents moyens d'observation serait la bienvenue.
\end{abstract}

\begin{abstract}
Near-field microscopies of biological macromolecules have shown an increasing growth of interest, but it should be kept in mind that such observations of biological material still pose a number of problems, at the levels of both sample preparation and image interpretation. An interdisciplinary approach is advised, for it could provide a combination of the traditional biophysical and biochemical methods, and that of electron microscopy, which are rather well established. Near-field microscopies, however, have unique possibilities to observe and analyse biological objects, which are not accessible to electron microscopy, and which offer through these new microscopies, a new dimension to biological imaging. Some examples illustrate the possibilities and limits of electron microscopy, in connection with which we propose some domains where a complementarity of these methods should be particularly opportune.
\end{abstract}

\section{Introduction.}

Near-field microscopes, either Atomic Force (AFM) or Scanning Tunnelling Microscopes (STM) are considered to have added a new dimension to biological research because of their capacity to provide nanometric resolution of objects which can be observed in air or liquid. These environments are more compatible with biological material than the vacuum and electron irradiation of 
electron microscopes. Even if these new machines provide a considerable amount of new information in materials science, their ability to image biological soft matter is still a subject of debate. Between the optimistic and pessimistic points of view, it must be recognized that some hopes and expectations were thwarted. However the numerous published observations reveal that these new methodologies could be very helpful in directions which were not necessarily envisaged at the outset. The recent considerable development of the optical near-field microscopes, with their spectroscopic capacities, especially when combined with STM and AFM, will probably offer far more information than that imagined a few years ago.

\section{Potential and limits of electron microscopy for the observation of biological macromolecules.}

Electron microscopy has the advantage of a long experience in the visualization of macromolecules since most of the problems related to their attachment to a support and their protection against vacuum and electron irradiation have been extensively studied and have more or less been solved within the limits of technical possibilities.

The observation of large proteins was rendered possible by using negative staining [reviewed in 1] which embeds them in an electron-dense film. This both provides contrast and protection against the aggressive environment of the microscope column. As this was not totally satisfactory, metal decoration on lyophilized preparations was developed and even adapted to the STM, especially on natural 2D structures used as a test material [2]. Cryo-electron microscopy was also developed to provide the molecules with a frozen aqueous environment, which preserves them at best [3,4]. All these techniques have been used extensively and have provided a considerable body of data of major importance in biology. All these methods, when associated with tilting experiments, offer a 3D reconstruction of the molecular architecture of favourable specimens, with the use of specialized statistical analysis programs (see for example [5]). In some exceptional situations, the observation of $2 \mathrm{D}$ crystals in cryo-electron microscopy can be studied by electron diffraction and has provided resolutions which are almost as good as those obtained with X-ray diffraction of actual 3D crystals of proteins (see for example $[6,7]$ ).

When proteins are too small to be visible (MW below $20 \mathrm{kDa}$ ), their oligomerization allows the visualization of repetitive motifs, which facilitate the description of the elementary particle in the polymer. These polymers can be linear or are able to built 2D arrangements.

When small proteins do not polymerize, the limit is attained and electron microscopy cannot be used efficiently. Fortunately, small molecules (MW below roughly $12 \mathrm{kDa}$ ) are accessible to structural analysis by Nuclear Magnetic Resonance (NMR), or can be crystallized, and their structure can be determined with these ultimate methods of structural analysis.

The visualization of nucleic acids and other filamentous molecules such as polysaccharides is also possible with a variety of spreading and contrasting methods (reviewed in [8]). However, the molecules are never perceived by themselves in any of these situations; their detection results from the use of a metal coating which decorates the molecules, or the use of a "stain" which consists in the physical adsorption of heavy metal clusters, and which indicates exactly where the molecules were on the support film, before their destruction by the electron irradiation. In spite of these drawbacks, the visualization of DNA molecules either embedded in cytochrome $c$ or detergent films, or adsorbed onto positively charged carbon support, has provided a considerable body of information which is complementary to that provided by the biochemical and biophysical approaches to the study of these molecules. In that field of investigation, electron microscopy possesses the unique capacity to visualize an unlimited number of individual molecules, and allows a statistical appraisal of the phenomena which can be detected at the level of their local conformation, or topology [9]. 
Nucleic acid-protein interaction is now an area of effervescent investigational activity in biology, since protein binding to nucleic acids seems to be the main mechanism of genomic expression and regulation. The development of molecular biology has provided biologists with unlimited means of synthesizing and modifying DNA and proteins involved in its metabolism. This has given rise to highly complex experimental possibilities which in turn have led to a better understanding of the structure and function of biological systems. Electron microscopy is able to depict DNAprotein interactions by showing, for instance, how a ligand binds to a known sequence on the DNA molecule and eventually modifies its local conformation, leading to complex structures which are involved in DNA replication, transcription, recombination, regulation of gene expression, etc... ([10-13], reviewed in [14]).

A wide variety of labels have been developed for the recognition and localization of these molecules for the "molecular biology of the cell". They make it possible to investigate the structure and the function of molecules in situ, at the ultimate degree of complexity which is present in cells. These labels, which are either radioactive, enzymatic or made of visible tags are easily detectable, even though their specificity and sensitivity continue to pose problems. They have evolved from large and more or less specifically bound markers to become tiny objects, such as gold spheres as small as $1-2 \mathrm{~nm}[15]$.

\section{Expectations and limits of near-field microscopies.}

The chief drawback with electron microscopy is that it is obliged to work on "dead" molecules. Hopes of working in buffered aqueous media with near-field microscopes appeared with the use of AFM. Its resolution capacities outdistanced that of electron microscopy, allowing the detection of atoms at the surface of mica, the support mostly used to visualize biological macromolecules in AFM.

Many publications have described the structure of different.macromolecules belonging to the nucleic acid and protein families, found to be accessible with AFM or STM. These papers presented either images of well-known molecules, or images of objects which were described as the molecules which were deposited on the support and imaged. The problems are the following: when known objects are imaged in AFM, they are usually clearly visible even if their width is exaggerated compared to that obtained with other methods, including electron microscopy. In addition, the resolution is worse than with electron microscopy, and the detection of surface details is still difficult or impossible. These phenomena have been discussed extensively under the name of "tip-object convolution", and progress has been achieved with sharper tips (Si instead of $\mathrm{Si}_{3} \mathrm{~N}_{4}$ tips), various types of "super tips", or with liquid medium (water or alcohol) for imaging, thus reducing mechanical problems [16]. The decrease in the apparent width of the molecules when sharper tips are used clearly indicates that the tip radius is often crucial, but theoretical analyses of various imaging modes of AFM (repulsion, attraction, friction, tapping, non-contact) show that the problem is not limited to the radius of the tip only. Since improvements are apparently feasible, hope of better images on soft biological material is conceivable.

When unknown molecules are imaged, it is often difficult to affirm that the objects displayed are a faithful representation of the molecules in question, because of probable conformational changes due to their attachment to the substrate, and also to the difficulties in interpreting images. Comparing observations obtained with electron microscopy and near-field microscopies could simplify these interpretations, but comparisons are seldom made.

Besides the advantage of operating in air or liquid media, AFM was faced with the need to firmly bind the specimen to the support. This can be solved with various techniques using either electrostatic binding or chemical bonding. This problem is even more difficult for STM investiga- 
tions, for which binding should be performed on conductive materials which are hydrophobic and do not bind biological macromolecules spontaneously. The insulating nature of biological material complicates the interpretation of STM images, when they can be obtained. Such limits with near-field microscopies must however be considered temporary since advances in the methodology and analysis of image formation are progressing rapidly. (See note added in proofs).

\section{Electron and near-field microscopies are complementary.}

Some examples could be proposed to illustrate the limitations of electron microscopy which could be surmounted with AFM or STM.

4.1 VISUALIZATION OF NUCLEIC ACIDS AND THEIR LIGANDS. - The initial objective of STM observation of nucleic acids, and particularly of double- stranded DNA, was to visualize the two strands of the double helix, and if possible to attempt to observe the small and large grooves, sites of numerous interactions with drugs [17-20] or proteins binding to DNA. AFM and STM have already demonstrated that they are complementary but only in the case of a few examples [16, 21]. With the visualization of single-stranded DNA, and the possibility of specifically recognizing the four bases, DNA could be sequenced. Even though specific images of these bases could be obtained when they are isolated from the DNA context [22], distinguishing them in situ on the phosphate-sugar backbone of DNA remains hypothetical, because such a helically arranged molecule poses complex spreading problems. Molecules bound to a support undergo considerable stress with the new microscopes as with electron microscopy, and numerous items must be analysed statistically before any definitive data can be presented. Although electron microscopy is to date unchallenged for the imaging of numerous objects, AFM and STM could efficiently provide topographic imaging of objects exhibiting tiny height modulations (e.g. small proteins or peptides bound to a specific DNA sequence). A prerequisite for the analysis of biological phenomena is the rapid attachment and easy screening of molecules at low magnification. When near-field microscopies become as efficient as electron microscopy in this respect, AFM and STM will surpass electron microscopy, because they cost less, are relatively easy to operate and their images are directly digitalized, which is of great help for data processing.

It should be borne in mind that these microscopes are potentially complementary since electron microscopy produces density images equivalent to X-ray radiographs, whereas AFM offers a topographic image, although this is also a subject of heated debate: AFM does not show the actual topography of the object, but in fact displays the movements of the tip, which result from complex interactions with the scanned surface of objects. This offers, however far more than a topographic information. The new AFM derivatives, which are sensitive to magnetism, electrochemical potentials, etc..., widen the dimensional horizons of these machines, especially in biology. Image interpretation could be expected to be increasingly complex and interesting, once all the phenomena which occur in image formation are fully understood. The same type of complexity, albeit different and perhaps more difficult, could be expected with STM on biological matter.

With these new developments, biologists will be confronted with further questions generated by these machines. The possibilities they now offer will engender new ideas for the development of specific labels which may be fundamentally different from those traditionally used in electron microscopy. Electron dense "stains", or high molecular weight labels used in transmission electron microscopy, which evolved with the advent of electron spectroscopic imaging are a case in point. New labels such as boron or lanthanides, not necessarily as dense as uranium, lead, gold, platinum, etc... have become accessible [23-25]. It can be envisaged that specially designed atoms or small molecules could be used to detect these labels specifically with specially adapted machines. 
Combining AFM or STM with near-field optical systems would allow the combination of topologic, fluorescent, magnetic etc... labels in the same machine, extending the limits of detection of various parameters on cells, chromosomes and molecules.

Another domain in which electron microscopy and especially cryo-electron microscopy is unequalled in its potential is the observation of nucleo-protein complexes, in which the topology and local conformations are particularly interesting. As already demonstrated by Dubochet and coworkers, 3D images of small DNA molecules embedded in ice can be obtained [26]. This method, rather than observation on a $2 \mathrm{D}$ substrate, is likely to provide fundamental results in the field of DNA-protein interactions.

4.2 VisUALIZATION OF PROTEINS AND OTHER MACROMOLECULES. - Filamentous molecules such as polysaccharides should be easily visualized with AFM or STM, as well as peptides of even artificially built systems. The association of chiral objects lead to chirally recognizable flat macrostructures, whose parameters are accessible to electron microscopy [27]. Such macromolecular devices, designed by Lehn and others, should provide interesting objects to image at high resolution with AFM or STM.

However, large proteins, which often have a globular shape, do not seem to be the material of choice to be studied with AFM or STM, unless surface details have to be shown, if they are accessible. Negative staining and cryo-electron microscopy are still the most appropriate techniques for these massive objects, whose structure can be deduced from tilted images [5].

Small proteins, with a recognizable shape or which oligomerize, could provide interesting objects for AFM and STM investigation for they are inaccessible to electron microscopic observation owing to their small size.

\section{Conclusion.}

This brief analysis indicates that biologists may need to use near-field microscopes to image macromolecules. The complementary use of the different techniques available should therefore be advocated. No one will challenge well-established techniques such as X-ray crystallography and NMR for the atomic resolution of biological molecules. Likewise, biochemical analysis will probably continue to have an edge over other methodologies in its appraisal of the structure of nucleic acids, and their interactions with various ligands.

Once again, it seems that the most efficient means of providing faithful images and significant results is to combine the various methodologies to both capitalize on their specific possibilities and via this combination seek to overcome their limits. The complexity of image formation must be adequately apprehended so that new interpretations become available and supersede the confines of the strictly topographic interpretation of AFM and even STM images.

In biology, special attention should be paid to avoid describing with AFM or STM, phenomena or structures already adequately depicted by electron microscopy. This does not mean that known structures which facilitate image interpretation and take into account tip effects are to be excluded. For example gold spheres are used to calibrate tips or test objects such as the classic circular plasmids or the tobacco mosaic virus are used to test the resolution capacity of microscopes. The colour pictures often employed in near-field microscopes offer an appreciable visual representation, but non-specialists do not always realize that these are pseudo-colours of not fully understood phenomena. In consequence, it is occasionally difficult for electron microscopists to show black and white pictures for they are perceived as outdated compared to their counterparts produced with "modern" microscopes. 
Most biological phenomena consist in an equilibrium between molecules which interact dynamically. The description of such phenomena must be based on the observation of numerous items which need to be analyzed statistically, even if only a few of them (which by chance correspond to the mean appearance of the population) are selected for publication.

It is vital that preparation procedures be developed for the global observation at low magnification of reproducibly and routinely disposed molecules to which zooming can be applied for the selection of the most significant images.

It is virtually impossible for biologists and physicists to keep abreast of the new developments in both near-field microscopes and molecular biology respectively. Thus interdisciplinary encounters between machine manufacturers, physicists and biologists are more than ever vital, if these disciplines are to develop harmoniously.

\section{Acknowledgements.}

M. Barray is thanked for the realization of the complex printing and mounting of the various pictures. We are indebted to our colleagues who collaborate with us for the realization of these original images which are issued from either published or unpublished results (N. Bonnet, C. Colliex, E. Nahon-Merlin, C. Mory, M. Tencé and F. Van Leuven). We also thank E. Paris (from Instrumat S. A., France) for using NanoScope III for the preparation of the AFM image, J.-C. Poulin for the STM image, and A. Kerrien (from Fondis Electronic, France) for imaging the metaphasic chromosomes with the TopoMetrix AFM. Lorna Saint-Ange is thanked for the extensive revision of the English text. The bibliographic references have been intentionally limited, with no attempt to cover the considerable amount of data on the use of near-field microscopes in biology.

Note added in proofs: Recent papers demonstrate the potentiality of AFM to analyse drug-DNA interactions [35], and that of the Tapping mode in liquid to visualize enzymes in action [36, 37]. The use of very low tunnelling currents now allows to image at a high resolution DNA molecules adsorbed onto mica [38]. These very important new results illustrate the rapid improvements of the techniques for biological applications.

\section{References}

[1] Harris J.R. and Horne R., Negative staining, in "Electron Microscopy in Biology. A practical approach", J.R. Harris Ed. (IRL Press, Oxford, 1991) 203-228.

[2] Wepf R., Amrein M., Bürkli U. and Gross H., Platinum/iridium/carbon: a high-resolution material for TEM, STM and SEM of biological macromolecules structures, J. Microsc. (Oxford) 163 (1991) 51-64.

[3] Dubochet J., Adrian M., Chang J.-J., Homo J.-C., Lepault J., McDowall A.W. and Schultz P., Cryoelectron microscopy of vitrified specimens, Quart. Rev. Biophys. 21 (1988) 129-228.

[4] Stewart M., Transmission electron microscopy of vitrified biological macromolecular assemblies, in "Electron microscopy in biology. A practical approach", J.R. Harris Ed. (IRL Press, Oxford, 1991) 229-242.

[5] Boisset N., Grassucci R., Penczek P., Delain E., Pochon F., Frank J. and Lamy J.-N., Three-dimensional reconstruction of a complex of human $\alpha 2$-Macroglobulin with monomaleimido nanogold $\left(\mathrm{Au}_{1.4 \mathrm{~nm}}\right)$ embedded in ice, J. Struct. Biol. 109 (1992) 39-45.

[6] Derosier D.J., Turn-of-the-century electron microscopy, Curr. Biol. 3 (1993) 690-692.

[7] Kühlbrandt W., Wang D.N. and Fujiyoshi Y., Atomic model of plant light-harvesting complex by electron crystallography, Nature 367 (1994) 614-621. 
[8] Delain E. and Le Cam E., The spreading of nucleic acids, in "Visualization of nucleic acids", G. Morel Ed. (CRC Press, 1995, in press).

[9] Muzard G., Théveny B. and Révet B., Electron microscopy of pBR322 DNA curvature. Comparison with theoretical models, EMBO J. 9 (1990) 1289-1298.

[10] Le Cam E., Théveny B., Mignotte B., Révet B. and Delain E., Quantitative electron microscopic analysis of DNA-protein interactions, J. Electron Microsc. Tech. 18 (1991) 375-386.

[11] Théveny B., Bailly A., Rauch C., Rauch M., Delain E. and Milgrom E., Association of DNA-bound progesterone receptors, Nature 329 (1987) 79-81.

[12] Le Cam E., Fack F., Ménissier-de Murcia J., Cognet J.A.H., Barbin A., Sarantoglou V., Révet B., Delain E. and De Murcia G., Conformational analysis of a 139 base pairs DNA fragment containing a single strand break, and its interaction with human poly (ADP-ribose) polymerase, J. Mol. Biol. 235 (1994) 1062-1071.

[13] Révet B.M.J., Sena E.P. and Zarling D.A., Homologous DNA targeting with recA protein-coated short DNA probes and electron microscope mapping on linear duplex molecules, J. Mol. Biol. 232 (1993) 779-791.

[14] Le Cam E. and Delain E., Nucleic acids-ligand interactions, in "Visualization of nucleic acids", G. Morel Ed. (CRC Press, 1995, in press).

[15] Hainfeld J.F. and Furuya F.R., A 1.4-nm gold cluster covalently attached to antibodies improves immunolabeling, J. Histochem. Cytochem. 40 (1992) 177-184.

[16] Delain E., Fourcade A., Poulin J.-C., Barbin A., Coulaud D., Le Cam E. and Paris E., Comparative observations of biological specimens, especially DNA and filamentous actin molecules in atomic force, tunnelling and electron microscopes, Microsc. Microanal. Microstruct. 3 (1992) 457-470.

[17] Butour J.-L., Delain E., Coulaud D., Le Pecq J.-B., Barbet J. and Roques B.P., Measurement of the expected DNA lengthening caused by mono- and bisintercalating drugs using electron microscopy, Biopolymers 17 (1978) 873-886.

[18] Révet B., Malinge J.M., Delain E., Le Bret M. and Leng M., Electron microscopic measurement of chain flexibility of poly(dG-dC) modified by cis-diamminedichloro-platinum(II), Nucleic Acids Res. 12 (1984) 8349-8362.

[19] Barcelo F., Muzard G., Mendoza R., Révet B., Roques B.P. and Le Pecq J.-B., Removal of DNA curving by DNA ligands. Gel electrophoresis study, Biochemistry 30 (1991) 4863-4873.

[20] Morier-Teissier E., Bernier J.-L., Coulaud D., Henichart J.-P. and Delain E., Electron microscopic observations of the effects of anthraquinone derivatives on plasmid DNA, J. Biomol. Struct. Dyn. 9 (1992) 653-666.

[21] Le Cam E., Fréchon D., Barray M., Fourcade A. and Delain E., Observation of binding and polymerization of Fur repressor onto operator-containing DNA with electron and atomic force microscopes, Proc. Natl. Acad. Sci. U.S.A. (1994) in press.

[22] Heckl W.M. and Holzrichter J.F., DNA base sequencing, Mol. Cryst. Liq. Cryst. Sci. Technol. [B]. Nonlinear Optics 2 (1992) 231-237.

[23] Bazett-Jones D.P., Electron spectroscopic imaging of chromatin and other nucleoprotein complexes, Electron Microsc. Rev. 5 (1992) 37-58.

[24] Delain E., Fourcade A., Révet B. and Mory C., New possibilities in the observation of nucleic acids by electron spectroscopic imaging, Microsc. Microanal. Microstruct. 3 (1992) 175-186.

[25] Bendayan M., Barth R. F., Gingras D., Londono I., Robinson P.T., Alam F., Adams D.M. and Mattiazzi L., Electron spectroscopic imaging for high-resolution immuno-cytochemistry: use of boronated protein A, J. Histochem. Cytochem. 37 (1989) 573-580.

[26] Bednar J., Furrer P., Stasiak A., Dubochet J., Egelman E.H. and Bates A.D., The twist, writhe and overall shape of supercoiled DNA change during counterion-induced transition from a loosely to a tightly interwound superhelix. Possible implications for DNA structure in vivo, J. Mol. Biol. 235 (1994) 825-847.

[27] Gulik-Krzywicki T., Fouquey C. and Lehn J.-M., Electron microscopic study of supramolecular liquid crystalline polymers formed by molecular-recognition-directed self-assembly from complementary chiral components, Proc. Natl. Acad. Sci. U.S.A. 90 (1993) 163-167.

[28] Delain E., Barray M., Tapon-Bretaudière J., Pochon F., Marynen P., Cassiman J.-J., Van der Berghe $H$. and Van Leuven F., The molecular organization of human alpha 2-Macroglobulin. An immunoelectron microscopic study with monoclonal antibodies, J. Biol. Chem. 263 (1988) 2981-2989.

[29] Boisset N., Taveau J.C., Barray M., Van Leuven F., Delain E. and Lamy J., Image analysis and three dimentional model of chymotrypsin-transformed human alpha 2- macroglobulin complexed with a monoclonal antibody specific for this conformation, Biol. Cell 64 (1988) 45-55. 
[30] Delain E., Pochon F., Barray M. and Van Leuven F., Ultrastructure of alpha 2- macroglobulins, Electron Microsc. Rev. 5 (1992) 231-281.

[31] Bonnet N. and Delain E., Digital acquisition and processing of STEM images of alpha 2-macroglobulin complexed with proteinases or zinc, J. Microsc. Spectrosc. Electron. 10 (1985) 505-514.

[32] Barbin A., Niveleau A., Dutrillaux B., Malfoy B. and Bourgeois C., In situ DNA methylation analysis of human tumor cells, mapping of methylcytosine-rich bands, Hum. Genet. 94 (1994) 684-692.

[33] Mory C., Colliex C., Révet B. and Delain E., Improved visualization of single- and double-stranded nucleic acids by STEM, Ultramicroscopy 7 (1981) 161-168.

[34] Nahon-Merlin E., Delain E., Coulaud D. and Lacour F., Electron microscopy of the reactions of antipolyA.polyU and anti-polyI.polyC antibodies with polynucleotide complexes and natural nucleic acids, Nucleic Acids Res. 8 (1980) 1805-1822.

[35] Hansma H.G., Browne K.A., Bezanilla M. and Bruice T.C., Bending and straightening of DNA induced by the same ligand: characterization with the atomic force microscope, Biochemistry 33 (1994) 84368441.

[36] Radmacher M., Fritz M., Hansma H.G. and Hansma P.K., Direct observation of enzyme activity with the atomic force microscope, Science 265 (1994) 1577-1579.

[37] Bezanilla M., Drake B., Kashlev M., Hansma P.K. and Hansma H.G., Motion and enzymatic degragation of DNA in the atomic force microscope, to be published.

[38] Guckenberger R., Heim M., Cevc G., Knapp H.F., Wiegräbe W. and Hillebrand A., Scanning tunneling microscopy of insulators and biological specimens based on lateral conductivity of ultrathin water films, Science 266 (1994) 1538-1540.

The following illustrations are a few examples of biological molecules for which interesting features have been revealed by electron microscopy. However this technique was not able to provide extensive information which could probably be acquired with complementary techniques such as cryo-electron microscopy or near-field microscopies. 
Fig. 1. - a) Gallery of complexes of two tetrameric alpha-2 macroglobulin molecules linked together by two antibody molecules (IgG F44 DC5) specific for a single epitope of the molecule: only two epitopes are recognized in these selected molecules [28-30]. Molecules are negatively stained with uranyl acetate and observed and photographed in the Scanning Transmission Electron Microscope (STEM) from the laboratory of "Physique des Solides" (Orsay) [31]. Neither the surface of the molecules nor their 3D arrangement are accessible with this technique. Scale $=50 \mathrm{~nm}$. b) Purified antibody (IgG) molecules observed after negative staining as in figure 1a with a Zeiss 902 in Electron Spectroscopic Imaging. These IgG are specific for 5-methylcytosine, the only base naturally modified in the human genome [32]. The inset shows the clear visibility of the three domains: one Fc and two Fab fragments. The technique does not allow a better resolution of the molecular structure and differentiation between these three domains. Scales $=50 \mathrm{~nm}$ and 15 $\mathrm{nm}$ for the inset. c) Plasmid DNA (pBR322) attached to a mica piece using $\mathrm{Ca}^{2+}$ to facilitate the binding [16]. The circular molecules are clearly visible after shadowing and observation in dark-field electron microscopy. Such a sample can be used as a control before observation in AFM on a similar but non-shadowed mica piece (see Fig. 2e). The same type of metal-plated molecules can be easily imaged in a STM (see Fig. 2f). Metallization allows easy visualization of several molecules at low magnification, but the inner structure of the double-stranded DNA molecule cannot be resolved. Scale $=100 \mathrm{~nm}$. d) Circular genome of the bacteriophage PM2 (length $3.2 \mu \mathrm{m}$ ). The DNA molecules are "stained" by the adsorption of uranium ions and visualized by annular dark-field imaging. The contour of the circular DNA molecule is clearly depicted, but the DNA molecule is not visible by itself; only the uranium atom clusters which remain attached to the carbon support indicate the presence of the molecule. No details are visible at the level of the double helix. Scale $=500 \mathrm{~nm}$. e) Portion of a double-stranded RNA molecule extracted fom the X virus of Drosophila and observed in dark-field mode with a STEM [33]. The RNA has been melted allowing the most fusible zones to be denatured and thus reveal the two elementary strands of the RNA. The size of the melted zones is about 120 base pairs long, corresponding to a length of $340 \AA$ (for a distance of $2.8 \AA$ between two adjacent base pairs in the RNA). As in the case of the preceding image, no detail can be seen except for the uranium clusters which remain linked to the separated or attached elementary strands. Scale $=5 \mathrm{~nm}$. $\mathrm{f}$ ) Plasmidic circular DNA complexed with a positively charged polymer (poly-L-lysine), and observed in dark-field after staining with uranyl acetate. As the DNA molecule is negatively charged the poly-L-lysine molecules stick to it and cause it to collapse gradually. Different stages of condensation can be visualized and eventually mapped. The sites of poly-L-lysine binding to DNA can be distinguished because of the thicker appearance of the DNA molecule which snaps back onto itself, leading to parallelly stuck double-stranded segments. It would be very interesting to be able to resolve both the polylysine and the DNA in these zones. Note that elementary filaments of poly-L-lysine are the limit of resolution of our preparation technique. Scale $=150$ nm. 


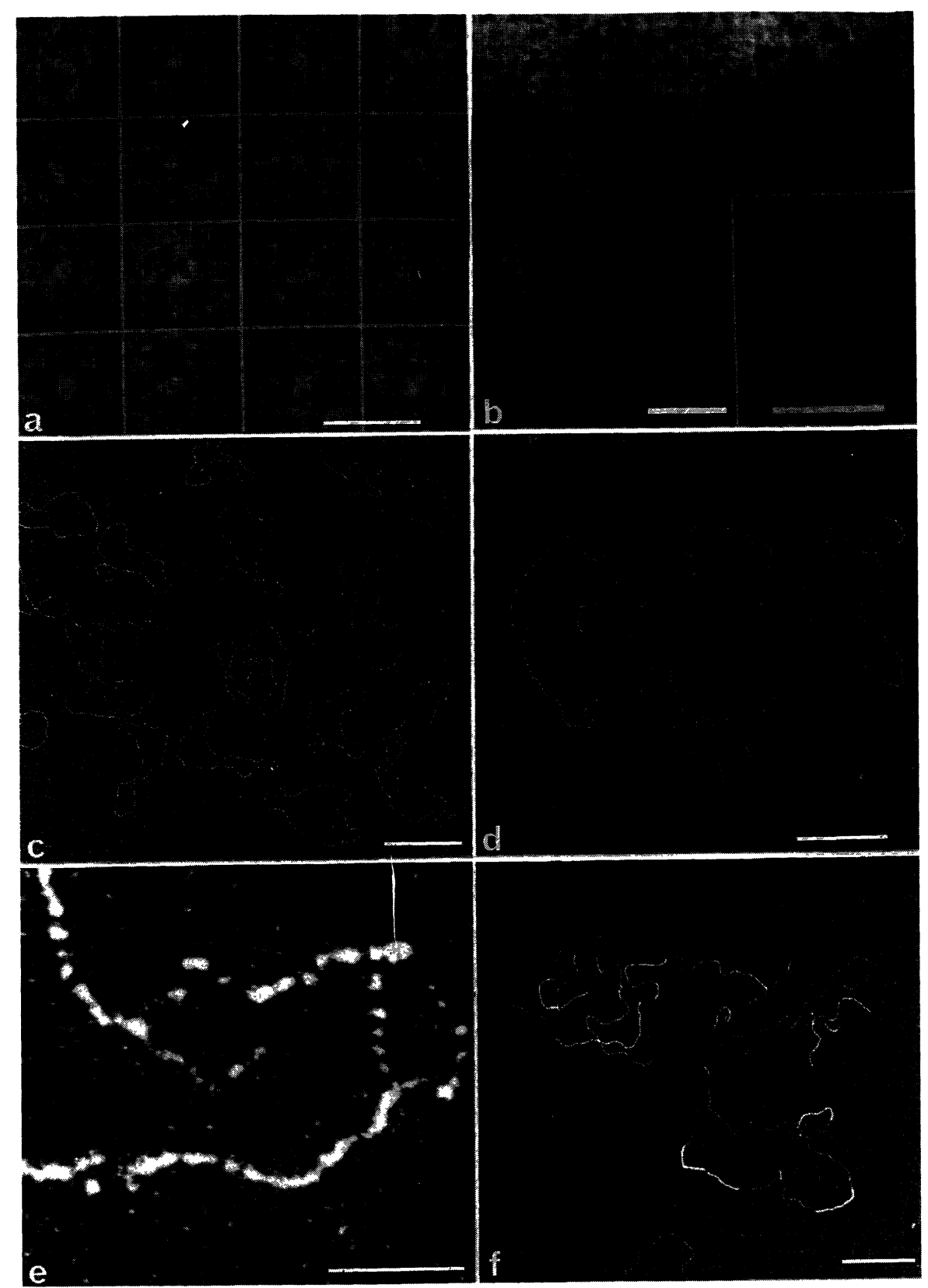

Fig. 1. - see caption on preceding page 


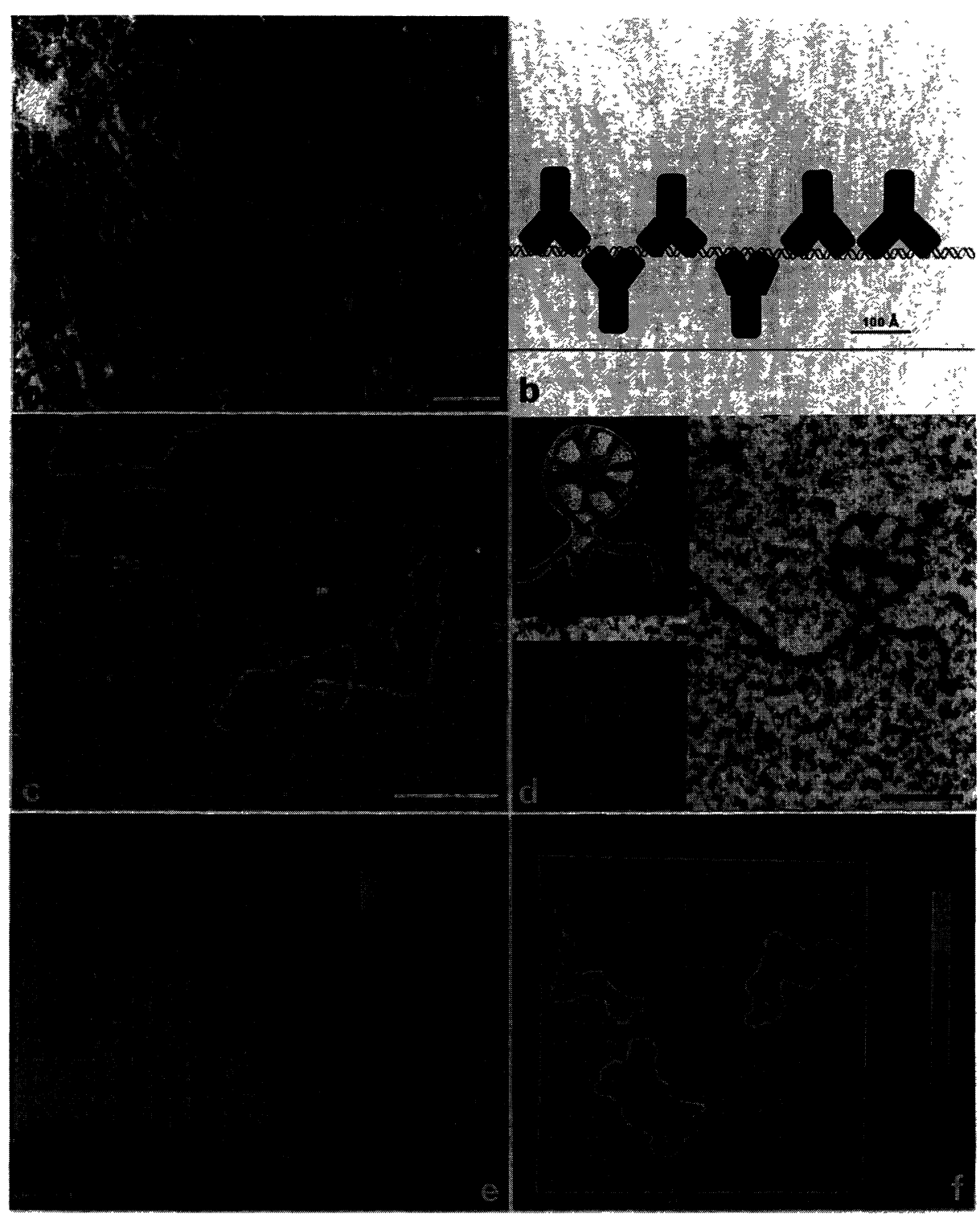

Fig. 2. - a) Tilted dark-field observation (Philips 300 ) of a tungsten-tantalum shadowing of immune complexes formed between double-stranded poly-ribonucleotides (RNA) and specific antibody molecules (IgG) [34]. Protruding from a complex aggregation of RNA and many IgG molecules, a long filament shows how the IgG can bind to it. The metal coating impedes clear visualization of the three domains of the IgG molecules. Scale $=80 \mathrm{~nm}$. b) From our images and crystallographic data on RNA and IgG, it is possible to draw (to scale) the way in which IgG can bind to the double-stranded RNA molecule. Near-field microscopes are expected to provide images with a resolution which will be close to that of the model. c) The same type of reaction can be observed in the same conditions as for figure 2a, which reveals the binding of IgM (a class of antibodies which look like five IgG elements). The preparation indicates that the IgM molecule can bind to the RNA via several elements (here 2 to 4). The two Fab fragments of each subunit of the IgM molecule are not resolved. Scale $=100 \mathrm{~nm}$. d) An IgM molecule can bind to a RNA molecule via all of its subunits, producing a wheel-shaped figure. A schematic drawing provides an interpretation and the scale of the figure, with an additional IgG molecule bound to the region where the RNA buckle is closed. Scale $=40 \mathrm{~nm}$. e) A large field of pBR322 plasmid circular DNA molecules adsorbed onto mica and imaged in a NanoScope III at low magnification in the "error" mode. The apparent thickness of the DNA molecule is exaggerated as described in [16]. f) STM image of three metal-coated pBR322 DNA molecules. Access to the fine molecular structure is impeded by the metal coating. 


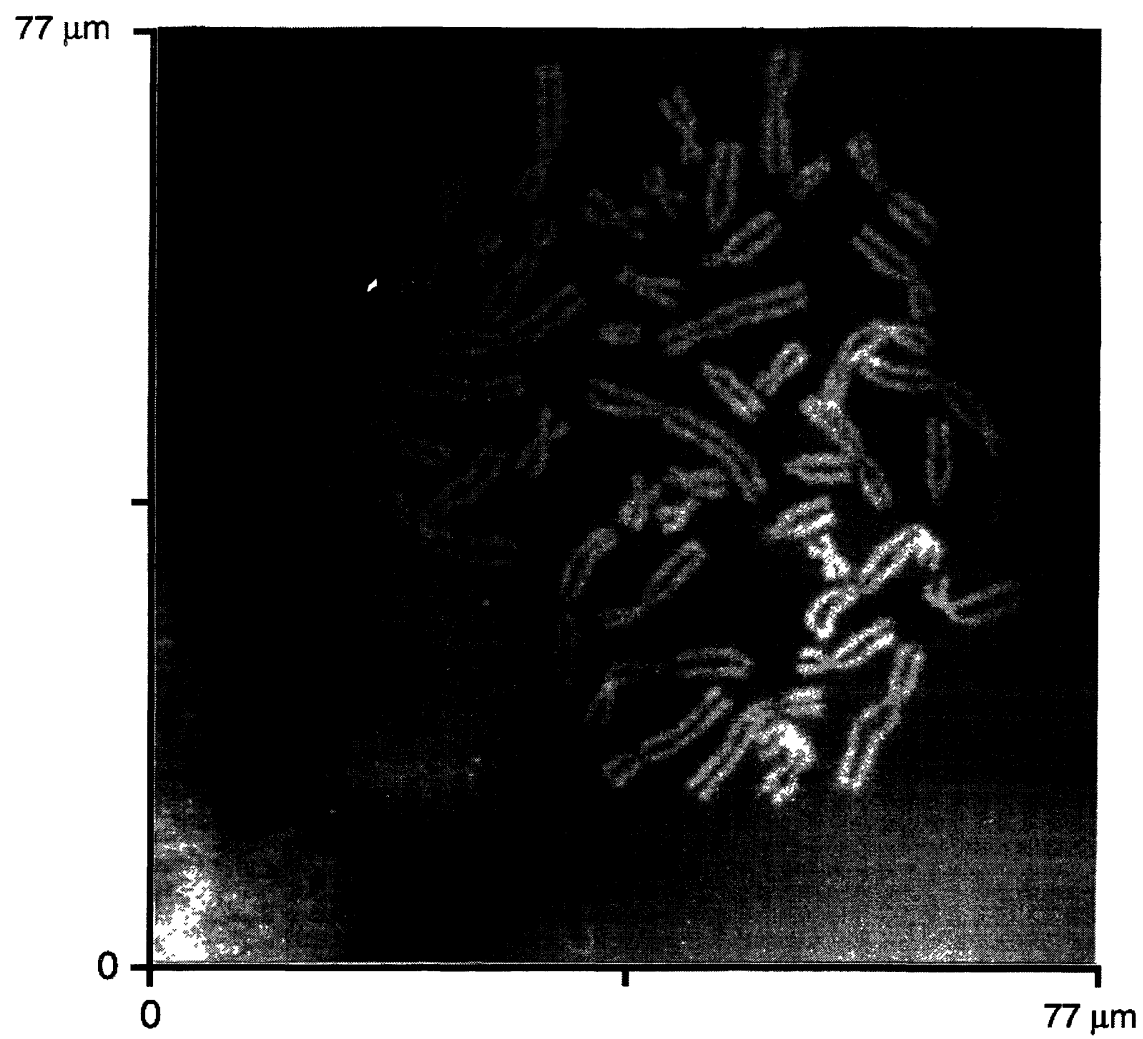

Fig. 3. - AFM image of human metaphasic chromosomes. This type ot image opens opportunities for high resolution observation of labelled specific sites on the chromosome, by detecting either the difference in height locally induced when IgG specifically recognizes a sequence or certain structures on DNA, or the fluorescent tags bound to IgG or other labels [32], using scanning optical near-field microscopes alone or in combination with AFM. 